\title{
Moduli stabilisation on the worldsheet
}

\author{
Jawad Arshad Deo, ${ }^{a}$ Neil Lambert ${ }^{b, 1}$ and Andreas Recknagel ${ }^{a}$ \\ ${ }^{a}$ Department of Mathematics, King's College London, \\ Strand, London WC2R 2LS, U.K. \\ ${ }^{b}$ CERN, Theory Division, \\ CH-1211 Geneva 23, Switzerland \\ E-mail: jawad.2.arshad@kcl.ac.uk, neil.lambert@cern.ch, \\ andreas.recknagel@kcl.ac.uk
}

ABSTRACT: We consider compactifications of type II string theory using exact internal CFT's with central charge $c=9+\epsilon,|\epsilon| \ll 1$, leading to an effective potential for the dilaton. For $\epsilon>0$ the potential is positive and the dilaton is ultimately driven to weak coupling. For $\epsilon<0$ the dilaton is driven to strong coupling, but we can stabilise the background by including D-branes. The resulting minimum admits an $A d S_{4}$ solution where the cosmological constant is of the order $\epsilon^{3}$ and the string coupling constant is of order $\epsilon$. Furthermore these CFT's typically do not possess any massless or tachyonic modes. Thus these vacua provide exact CFT descriptions of moduli stabilisation in weakly coupled string theory.

KEywords: Conformal Field Models in String Theory, Superstring Vacua, Flux compactifications

ARXIV EPRINT: 1012.3972

\footnotetext{
${ }^{1}$ On leave of absence from King's College, London U.K.
} 


\section{Contents}

1 Introduction 1

2 Central charge deficit and four-dimensional effective action 2

$\begin{array}{lll}3 & \text { The external sector } & 6\end{array}$

4 The internal sector: epsilon-Gepner models 48

5 Some examples, and spectra of light fields 11

6 Conclusions and open problems 14

A 'Best' $\epsilon>0$ models $\quad 15$

$\begin{array}{ll}\text { B Examples of models } & 16\end{array}$

\section{Introduction}

Compactification of the 10-dimensional target space of superstring leads to a vast choice for the data describing the internal compact manifold. Often, especially in supersymmetric compactifications, there are massless moduli fields that describe the deformations of the internal manifold. A fundamental problem in string phenomenology therefore is to find ways to construct vacua without any moduli since there are strong cosmological constraints on the existence of massless or very light scalar fields. A solution to this problem has been devised over the past few years in the form of flux compactifications (see [18] for a review). While they can preserve some supersymmetry, fluxes give vacuum expectation values to the massless fields and hence stabilise the moduli. According to current wisdom this leads to a large number of vacua known as the 'landscape' since fluxes can take many different discrete values.

These models have been largely studied using effective field theory and supergravity. This requires that the compactification scale is large compared to the string scale but on the other hand is small compared to experimentally accessible scales. From the worldsheet point of view this approach seems somewhat contrived. Therefore it is of interest to consider compactifications of string theory using internal CFT's which do not necessarily have a large geometric limit. The classic example of CFT compactifications are Gepner Models [9]. These are typically thought of as CFT analogues of compact Calabi-Yau manifolds whose size is of order the string scale, however they are formulated without recourse to geometric data. The construction of Gepner Models involves taking a tensor product of minimal models whose central charges add up to 9 . The models that we discuss here carry the 
correct overall central charges $c=15$, but the decomposition differs from the usual $6+9$ split for the internal and external sectors. Instead we take our external theory to be slightly non-flat with $c_{\text {ext }}=6-\epsilon$ and hence $c_{\text {int }}=9+\epsilon$, with $|\epsilon| \ll 1$ (other discussions of nongeometric compactifications and moduli stabilisation include [15]). We also note that for $\epsilon>0$ similar models were studied long ago within the context of cosmology $[3,5,6]$, however here we wish to emphasise the issue of moduli stabilisation.

We will see that there are plenty of explicit internal compact unitary CFT's with $|\epsilon| \ll 1$. For $\epsilon<0$ there is no limit to how small $\epsilon$ can be. However for $\epsilon>0$ this does not seem to be the case and the best models that we have found have $\epsilon \sim 10^{-6}$. Keeping $\epsilon$ small means that the theory remains weakly coupled and the external curvatures are small so one recovers a reliable four-dimensional effective field theory. Furthermore models can be obtained such that the spectrum of the full theory is free of massless fields. The exact spectrum is model-dependent but we discuss general characteristics shared by all models constructed in the prescribed way. Although in this note we will not seek to obtain a realistic spectrum of massless fields, we hope that this can be addressed in future work.

The rest of this paper is organised as follows. In section 2 we discuss the over-all set-up of our models and derive the four-dimensional effective field theory. We use this to argue that, for $\epsilon<0$ we can introduce D-branes that lead to a stabilising potential for the dilaton. This mechanism also appeared in [31] in the context of orientifolds of non-critical string theory. The resulting minimum admits an $A d S_{4}$ vacuum state at weak coupling and weak curvature. In section 3 we outline the linear dilaton CFT which can be used as an exact background for the external part. In section 4 we give a brief review of the construction of Gepner models and their D-branes, extending this to the case where the total central charge is $9+\epsilon$. In section 5 we discuss the spectrum of some explicit examples. Section 6 is our conclusion. We also list some examples of the internal CFT's that can be used in the appendix.

\section{Central charge deficit and four-dimensional effective action}

The sigma-model action of the string moving in a background with massless fields is given by,

$$
\begin{aligned}
S=-\frac{1}{4 \pi \alpha^{\prime}} \int d^{2} \sigma \sqrt{\gamma}\{ & \gamma^{\alpha \beta} g_{\mu \nu}(X) \partial_{\alpha} X^{\mu} \partial_{\beta} X^{\nu} \\
& \left.+\epsilon^{\alpha \beta} b_{\mu \nu}(X) \partial_{\alpha} X^{\mu} \partial_{\beta} X^{\nu}+\alpha^{\prime} \mathcal{R} \phi(X)\right\},
\end{aligned}
$$

where $\alpha, \beta=0,1, \mu, \nu=0,1, \ldots, D-1, \mathcal{R}$ is the Ricci scalar of the worldsheet metric $\gamma_{\alpha \beta}$. The string coupling constant is $g_{s}=e^{\phi}$ and hence string perturbation theory is valid where ever $g_{s}$ is small. Although this action is classically conformally invariant this is generically broken in the quantum theory. To one-loop order one finds the conditions for conformal invariance are $\beta_{\mu \nu}^{g}=\beta_{\mu \nu}^{b}=0$ where

$$
\begin{aligned}
& \beta_{\mu \nu}^{g}=\alpha^{\prime}\left(R_{\mu \nu}-\frac{1}{4} H_{\mu \lambda \rho} H_{\nu}^{\lambda \rho}+2 \mathcal{D}_{\mu} \mathcal{D}_{\nu} \phi\right)+\mathcal{O}\left(\alpha^{\prime 2}\right) \\
& \beta_{\mu \nu}^{b}=\alpha^{\prime}\left(\mathcal{D}^{\lambda} H_{\mu \nu \lambda}-2 \mathcal{D}^{\lambda} \phi H_{\mu \nu \lambda}\right)+\mathcal{O}\left(\alpha^{\prime 2}\right)
\end{aligned}
$$


If these equations are satisfied one finds that the central charge $c$ of the worldsheet conformal field theory is indeed a constant and is given by

$$
c=\frac{3}{2} \beta^{\phi}
$$

where

$$
\beta^{\phi}=D+\alpha^{\prime}\left(4(\mathcal{D} \phi)^{2}-4 \mathcal{D}^{2} \phi-R+\frac{1}{2 \cdot 3 !} H^{2}\right)+\mathcal{O}\left(\alpha^{\prime 2}\right) .
$$

To construct string backgrounds, one needs a worldsheet CFT with $c=15$. Traditionally this is achieved by taking $D=10$ and solving $\beta_{\mu \nu}^{g}=\beta_{\mu \nu}^{b}=0$ and $\beta^{\phi}=10$ at lowest order. For weakly curved backgrounds, that is curvatures that are large compared to the string scale, this is a good approximation and the resulting equations of motion arise from the supergravity spacetime effective action. Furthermore we wish to take four dimensions to be large, nearly flat, and the remaining ones to be compact. For the $\alpha^{\prime}$ expansion to be valid however the compact space must be smooth and have a length scale that is large compared with $\sqrt{\alpha^{\prime}}$.

A way to avoid this is to consider string backgrounds which are a direct product of an internal, exact, CFT with $c_{\text {int }}=9$ and a non-compact sigma model with a four-dimensional flat target space and hence $c_{\text {ext }}=6$. A class of exact CFT's with $c_{\text {int }}=9$ are provided by the so-called Gepner models and these are in turn constructed as a tensor product, with identifications, of minimal models, as discussed below.

Here we will try something slightly different. We will consider the CFT to be a tensor product of a sigma model with $D=4$ with central charge 6 and an internal CFT with central charge $c_{\text {int }}=9+\epsilon$ where $\epsilon$ is a small dimensionless number, possibly negative. As a result we see that the conditions for conformal invariance of the non-compact sigma model are

$$
4-\frac{2 \epsilon}{3}=4+\alpha^{\prime}\left(4(\mathcal{D} \phi)^{2}-4 \mathcal{D}^{2} \phi-R+\frac{1}{2 \cdot 3 !} H^{2}\right)+\mathcal{O}\left(\alpha^{\prime 2}\right)
$$

where the first equation comes from the condition $c=c_{\text {ext }}+c_{\text {int }}=15$. From this equation we see that $\alpha^{\prime} M^{2} \sim \epsilon$, where again $M$ is the scale of the curvature of the background. Provided that $|\epsilon| \ll 1$ then the perturbation expansion is still valid, indeed we can think of the $\alpha^{\prime} M^{2}$ expansion as an expansion in $\epsilon$. We call $\epsilon$ the central charge deficit following $[5,6]$ and our goal will be to search for models with small $\epsilon$. We take the external theory to be in $D=4$.

The background equations of motion (2.2a), (2.2b), (2.5) can be derived as the EulerLagrange equations of the spacetime effective action

$$
S_{\text {eff }}=\frac{1}{\alpha^{\prime}} \int d^{4} X \sqrt{-g} e^{-2 \phi}\left(R+4(\mathcal{D} \phi)^{2}-\frac{1}{2 \cdot 3 !} H^{2}-\frac{2 \epsilon}{3 \alpha^{\prime}}\right)+\ldots,
$$

where again the ellipsis denotes higher order terms in $\alpha^{\prime}$ and derivatives, i.e. $\epsilon$. To proceed it is useful to go to so-called Einstein frame

$$
\tilde{g}_{\mu \nu}=e^{-2 \phi} g_{\mu \nu}
$$

so that, in terms of $\tilde{g}_{\mu \nu}$ the spacetime action is

$$
\tilde{S}_{\text {eff }}=\frac{1}{\alpha^{\prime}} \int d^{4} X \sqrt{-\tilde{g}}\left(\tilde{R}-2(\mathcal{D} \phi)^{2}-\frac{1}{2 \cdot 3 !} e^{-2 \phi} H^{2}-\frac{2 \epsilon}{3 \alpha^{\prime}} e^{2 \phi}\right)+\cdots
$$




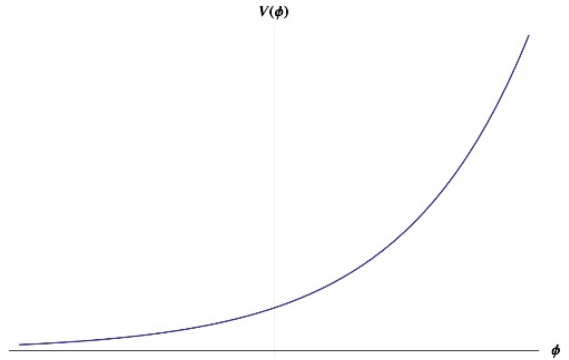

(a) $\epsilon>0$

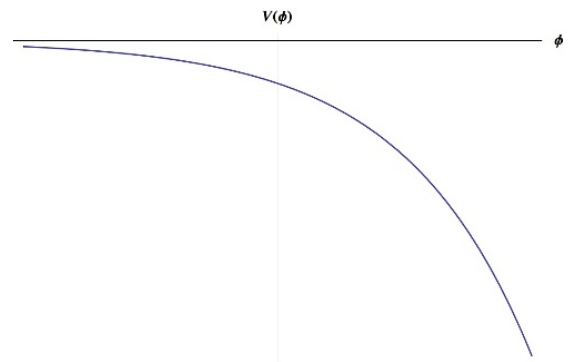

(b) $\epsilon<0$

Figure 1. Effective dilaton potentials in the Einstein frame.

where all quantities with tilde are in Einstein frame. Here we find an effective dilaton potential

$$
\tilde{V}(\phi)=\frac{2 \epsilon}{3 \alpha^{\prime}} e^{2 \phi}
$$

see figure 1. We will be interested in cosmological solutions that are consistent with an FRW universe. In this case, it is helpful to dualise he NS-NS Kalb-Ramond field into an axion $a$ via

$$
H_{\mu \nu \lambda}=\varepsilon_{\mu \nu \lambda \rho} e^{2 \phi} \partial^{\rho} a
$$

which accounts for another massless modulus. Thus we have

$$
\tilde{S}_{\text {eff }}=\frac{1}{\alpha^{\prime}} \int d^{4} X \sqrt{-\tilde{g}}\left(\tilde{R}-2(\mathcal{D} \phi)^{2}-\frac{1}{2} e^{2 \phi}(\mathcal{D} a)^{2}-\frac{2 \epsilon}{3 \alpha^{\prime}} e^{2 \phi}\right)+\ldots,
$$

In addition to these universal string fields that arise from the NS-NS ground state of the string, a generic compactification will also lead to other light states, i.e. scalar field whose mass is small compared to $1 / \sqrt{\alpha^{\prime}}$. Thus the total effective action is

$$
\tilde{S}=\tilde{S}_{\mathrm{eff}}-\frac{1}{2 \alpha^{\prime}} \sum_{a} \int d^{4} x \sqrt{-\tilde{g}}\left(\partial_{\mu} \chi_{a} \partial^{\mu} \chi_{a}+e^{2 \phi} m_{a}^{2} \chi_{a}^{2}\right)
$$

where the $\chi_{a}$ represent the other light scalar fields and $m_{a}$ is the string-frame mass, as computed in the CFT. Later in the text, we relate this to the conformal dimension in the test case of an exact background containing linear dilaton in the external, noncompact sector.

For $\epsilon>0$ we find that the dilaton will ultimately run to weak coupling; $\phi \rightarrow-\infty$. However there will be solutions where $\phi$ initially runs up the potential, stops, and then rolls back down to $\phi \rightarrow-\infty$. At the turning point $V(\phi)>0$ with $\dot{\phi}=0$ and hence there will be a short period of inflation as in the model of [22] (only here it is realised within perturbative string theory).

For large $\phi$ the strings are strongly coupled and $\epsilon$ cannot be used to suppress the curvatures and our $\alpha^{\prime}$ expansion isn't valid. For $\epsilon>0$ this region can be avoided. However, if $\epsilon<0$ then we see that eventually $\phi \rightarrow \infty$ and we always end up in the uncontrolled regime. Suppose that we introduce D-branes into the background which are extended along the four non-compact spatial directions. Such a brane would introduce a term in the effective potential of the form

$$
S_{\text {brane }}=-T \int d^{4} x \sqrt{-g} e^{-\phi}
$$




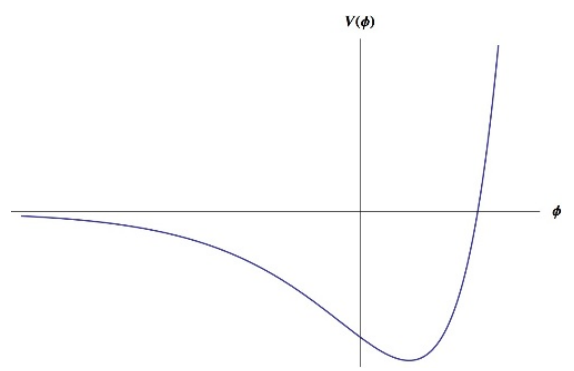

Figure 2. Dilaton potential for $\epsilon<0$, stabilised by a space-filling brane.

with $T>0$ and again we are in the string frame. Transforming to the Einstein frame gives

$$
\tilde{S}_{\text {brane }}=-T \int d^{4} x \sqrt{-\tilde{g}} e^{3 \phi}
$$

Combining this with the charge deficit contribution we arrive at the effective dilaton potential

$$
\tilde{V}(\phi)=\frac{2 \epsilon}{3 \alpha^{\prime}} e^{2 \phi}+T e^{3 \phi}
$$

see figure 2. This potential has an extremum at

$$
\frac{4 \epsilon}{3 \alpha^{\prime}} e^{2 \phi}+3 T e^{3 \phi}=0
$$

which, for positive tension branes $(T>0)$, has a solution if $\epsilon<0$;

$$
e^{\phi_{0}}=-\frac{4 \epsilon}{9 T \alpha^{\prime}}
$$

with

$$
\tilde{V}\left(\phi_{0}\right)=\frac{32 \epsilon^{3}}{9^{3} \alpha^{\prime 3} T^{2}}<0
$$

One can see that this is in fact a stable minimum. For small and negative $\epsilon$ this critical point is weakly coupled with a small but negative cosmological constant, see also [31].

In the rest of this paper we will develop the worldsheet CFT's required in these constructions. We first consider the case $\epsilon>0$ where the running of the dilaton down the potential can be modeled exactly by a timelike linear dilaton CFT (although there are of course other solution whose exact CFT is unkown). For the $\epsilon<0$ case we need to construct the internal compact CFT and also find suitable D-branes that can be introduced to stabilise the dilaton. We will see that models can readily be found with small $\epsilon$ and no massless fields. In such cases there are no problems with tadpoles from the D-branes and thus they can be consistently incorporated into the background. Furthermore the absence of massless field implies that the only massless moduli left is the axion $a$. However typically non-perturbative such effects, such as worldsheet instantons, produce a periodic potential for $a$ which must therefore have a global minimum. Thus these models have an $A d S_{4}$ vacuum with no massless fields.

In the following, we will present examples of CFTs that exhibit non-zero central charge deficits in the external and internal sector. One should keep in mind, however, that the 
non-trivial dilaton potential in principle affects the "trustworthiness" of these CFT backgrounds. For $\epsilon>0$, the dilaton is driven towards $\phi_{0}=-\infty$, thus string loops effects are switched off and the tree-level CFT may be regarded as a complete description of the background. For $\epsilon>0$, on the other hand, adding a brane makes the dilaton settle at a small but finite value of $g_{S}$; thus the tree-level is not the full story, and loop corrections (as well as backreactions of the brane) will play a role. At the minimum of the dilaton potential, the theory should in principle be described by some new CFT taking these corrections into account, and due to the negative cosmological constant $V\left(\phi_{0}\right)$, it is natural to suspect this CFT to be realised by a sigma model with an $A d S_{4}$ target space. However a main benefit of our construction is that the derivative expansion turns out to be an expansion in $\epsilon$ and hence the leading order contributions to the effective theory can be very accurate. Thus in these cases we don't expect the exact solution to differ from that obtained from the effective action in any substantial way.

\section{The external sector}

The external CFTs we will use here to build string backgrounds are linear dilaton models. They can be described as sigma models with the bosonic part of the action given in (2.1), where one sets $\phi(X)=V_{\mu} X^{\mu}$ for some fixed vector $V_{\mu}$ in four-dimensional Minkowski space. The main piece of information we will need in the following section is the mass shell formula arising from the physical state condition in string theory, see below.

In the supersymetric version, the bosons $X^{\mu}$ are accompanied by free fermions $\psi^{\mu}$, and the generating fields of the (left-moving) $N=1$ superconformal algebra can be written as

$$
\begin{aligned}
T_{\text {ext }}(z) & =-\frac{1}{\alpha^{\prime}}: \partial X_{\mu} \partial X^{\mu}:+V_{\mu} \partial^{2} X^{\mu}-\frac{1}{2} \psi_{\mu} \partial \psi^{\mu}, \\
G_{\text {ext }}(z) & =i\left(2 / \alpha^{\prime}\right)^{\frac{1}{2}} \psi_{\mu} \partial X^{\mu}-i\left(2 \alpha^{\prime}\right)^{\frac{1}{2}} V_{\mu} \partial \psi^{\mu}
\end{aligned}
$$

The central charge of the Virasoro algebra is, in $D=4$ dimensions,

$$
c_{\mathrm{ext}}=6+6 \alpha^{\prime} V^{2}
$$

so unless $V_{\mu}$ is lightlike, the linear dilaton can serve as the external CFT in string compactifications with non-trivial central charge deficit. $V_{\mu}$ is constrained by the choice we will make for $\epsilon$ in the internal CFT,

$$
\epsilon=-6 \alpha^{\prime} V_{\mu} V^{\mu}
$$

In many respects, the linear dilaton is close to a free boson CFT, the main difference being the $V_{\mu}$-term in $T_{\text {ext }}(z)$. As a consequence, the field $\partial X^{\mu}$ is not a conserved current, instead there is a so-called background charge, and correlation functions - which will not concern us in this paper - would have to be computed using screening operators, see $[7,8]$ and also $[5,6]$.

The fields we are interest in for string theory purposes are the vertex operators

$$
\mathcal{V}_{p}=: e^{i p_{\mu} X^{\mu}}:
$$


which are primary with conformal dimension

$$
h_{p}=\frac{\alpha^{\prime}}{4}\left(p_{\mu} p^{\mu}+2 i p_{\mu} V^{\mu}\right) .
$$

In order to analyse spectra of string compactifications, one starts from the physical state condition

$$
\left.\left(L_{0}^{\text {tot }}-a\right) \mid \text { phys }\right\rangle=0
$$

to extract a formula for the mass of a string state in terms of conformal dimensions. $a$ is a normal-ordering constant. We tacitly assume that $\mid$ phys $\rangle$ is a primary state for the super-Virasoro algebra to begin with.

$L_{0}^{\text {tot }}$ is a sum of the internal $L_{0}^{\text {int }}$ and of the zero mode of the linear dilaton energymomentum tensor which acts as

$$
L_{0}^{\mathrm{ext}}=\frac{\alpha^{\prime}}{4} p_{\mu} p^{\mu}+i \frac{\alpha^{\prime}}{2} V_{\mu} p^{\mu}+N .
$$

Here, the offset $N$ from the conformal dimension (3.5) accounts for modes $L_{n}^{\text {ext }}$ or $\psi_{r}^{\mu}$ applied to ground states $|p\rangle$; we have $N \in \frac{1}{2} \mathbb{Z}_{+}$in the Neveu-Schwarz sector and $N \in \mathbb{Z}_{+}$in the Ramond sector.

Inserting this into the physical state condition, we get

$$
\frac{\alpha^{\prime}}{4} p_{\mu} p^{\mu}+i \frac{\alpha^{\prime}}{2} V_{\mu} p^{\mu}=\Delta_{N, a} \quad \text { with } \quad \Delta_{N, a}=a-N-h_{\text {int }}
$$

and see that we need to admit complex $p^{\mu}=\rho^{\mu}+i \sigma^{\mu}$ in order to have non-trivial solutions - assuming that the conformal dimensions $h_{\text {int }}$ from the internal CFT are real. The imaginary part of the equation,

$$
\frac{\alpha^{\prime}}{2} \rho_{\mu}\left(\sigma^{\mu}+V^{\mu}\right)=0
$$

is solved by fixing $\sigma_{\mu}=-V_{\mu}$. Inserting this into the real part and using (3.3), we can rewrite the physical state condition in terms of the charge deficit $\epsilon$ as

$$
\frac{\alpha^{\prime}}{4} \rho_{\mu} \rho^{\mu}=\Delta_{N, a}+\frac{\epsilon}{24} .
$$

Some interpretational issues remain. First of all, we have to relate the spacetime mass of a string state to the quantities in this physical state condition. We will set

$$
m^{2} \equiv-\frac{\alpha^{\prime}}{4}\left(p_{\mu} p^{\mu}+2 i V_{\mu} p^{\mu}\right)=-\Delta_{N, a}
$$

This choice is motivated by considering the coupling of the linear dilaton to a massive scalar field [4] with the action given by

$$
-\frac{1}{2 \alpha^{\prime}} \int d^{4} x \sqrt{-g} e^{-2 \phi}\left\{(\partial \chi)^{2}+m^{2} \chi^{2}\right\}
$$

whose equation of motion gives the mass-shell condition

$$
p_{\mu} p^{\mu}+2 i V_{\mu} p^{\mu}+m^{2}=0 .
$$


Thus we can identify $m^{2}$ in the effective action with the mass-squared computed by the conformal dimension of the internal CFT via (3.10). By computing quantum fluctuations around the linear dilaton background in the weak gravity limit, we can see that the dilaton and the graviton remain massless, even when the background affects the physical mass via the background vector $V_{\mu}$ in the physical state condition.

In the paper $[5,6]$, the other natural choice $m^{2}=-\frac{\alpha^{\prime}}{4} \rho_{\mu} \rho^{\mu}$ was used, which leads to an $\epsilon$-dependent mass shift of the whole spectrum due to the presence of the linear dilaton. We will briefly come back to this choice in the next section, but let us point out right away that the most important qualitative feature of our string compactifications does not depend on which of the two definitions of spacetime mass we use: either way, we will encounter many models that have no massless moduli. Physically the difference arises because the background geometry is not flat and hence the solution to a massive wave equation can behave with a different mass from that which appears in the Lagrangian.

The second "interpretational" issue concerns the normal ordering constant $a$ in the physical state condition. Here, we will make the standard choices

$$
a_{\mathrm{NS}}=\frac{1}{2}, \quad a_{\mathrm{R}}=\frac{1}{2}-\frac{d}{16}
$$

for the NS and R sectors respectively, where $d / 16$ is the contribution coming from $d=D-2$ spin fields in the transverse direction. This leads to $a_{\mathrm{R}}=\frac{3}{8}$ for our $D=4$ linear dilaton theory.

The work [1], on the other hand, introduces an $\epsilon$-shift into the normal ordering constant. There the shift arises from solving the light-cone gauge constraint, after having introduced non-standard $V_{\mu}$-dependent Lorentz group generators, which allow to "restore" the Lorentz symmetry which is, at the face of it, broken by the linear dilaton theory. The effect of the shift in $a$ on the physical state condition is as switching between the above definitions of mass.

\section{The internal sector: epsilon-Gepner models}

In [9], Gepner introduced algebraic string compacfications using exact CFTs instead of sigma-models on compact Calabi-Yau manifolds for the internal sector of the worldsheet theory. Gepner's internal CFTs are tensor products of minimal models of the $N=2$ super Virasoro algebra, such that their central charges add up to $15-3 D / 2$ where $D$ is the dimension of the non-compact spacetime. The full theory is then a (GSO-projected) tensor product with an external CFT.

The string backgrounds discussed here, with non-vanishing central charge deficit $\epsilon$, are formed in close parallel to Gepner models, therefore we review the main building blocks of Gepner's construction.

The internal sector is comprised of minimal models of the $\mathcal{N}=2$ superconformal algebra; these are cosets

$$
\frac{\mathrm{SU}(2)_{k} \times \mathrm{U}(1)_{4}}{\mathrm{U}(1)_{2 k+4}}
$$


where the subscripts denote the levels of the affine Lie algebras; the central charge is

$$
c(k)=\frac{3 k}{k+2}, \quad k=1,2, \ldots .
$$

A minimal model is a rational CFT, whose finitely many irreducible representations (of the bosonic subalgebra) can be labeled by $(l, m, s)$, where $l$ refers to the $\mathrm{SU}(2)_{k}, s$ to the $\mathrm{U}(1)_{4}$ in the numerator, and $m$ to the $\mathrm{U}(1)_{2 k+4}$ in the denominator. These labels satisfy

$$
\begin{aligned}
& l=0,1, \ldots, k, \quad m=-k-1,-k, \ldots, k+2 \quad s=-1,0,1,2, \\
& \text { with } \quad l+m+s \text { even. }
\end{aligned}
$$

Representations with $s=0,2$ belong to the NS sector, while those with $s= \pm 1$ to the R-sector. Triples $(l, m, s)$ and $(k-l, m+k+2, s+2)$ give rise to the same representation and are identified. The conformal dimension $h$ and U(1)-charge $q$ of the highest weight state with labels $(l, m, s)$ are given by

$$
h_{m, s}^{l}=\frac{l(l+2)-m^{2}}{4(k+2)}+\frac{s^{2}}{8}, \quad q_{m, s}^{l}=\frac{m}{k+2}-\frac{s}{2}
$$

as as long as the labels $(l, m, s)$ lie in the so-called standard range, i.e. they satisfy

$$
l=0,1, \ldots, k, \quad|m-s| \leq l, \quad s=-1,0,1,2, \quad l+m+s \text { even }
$$

or

$$
l=1,2, \ldots, k, \quad m=-l, \quad s=-2 .
$$

Every tuple $(l, m, s)$ may be brought into the standard range using the transformations,

$$
(l, m, s) \mapsto(l, m+2 k+4, s), \quad(l, m, s) \mapsto(l, m, s+4) .
$$

Representations can be grouped into pairs $(l, m, s)$ and $(l, m, s+2)$, each such pair makes up a full $\mathcal{N}=2$ super Virasoro module; all states in the same bosonic sub-representation have the same fermion number (or U(1)-charge) modulo two.

A Gepner model is formed from a tensor product of $r$ minimal models with central charges $c\left(k_{i}\right)$ together with $D$ external free bosons and fermions. The latter make up an $\mathrm{SO}(d)_{1}$ current algebra, where $d=D-2$ (working in the light cone gauge). In Gepner's original construction, the levels $k_{i}$ are chosen such that

$$
c_{\text {int }} \equiv \sum_{i=1}^{r} c\left(k_{i}\right)=15-\frac{3}{2} D,
$$

For compactifications to $D=4$ dimensions, we must have $3<r \leq 9$ because $1 \leq c\left(k_{i}\right) \leq 3$; the most famous example is $(3,3,3,3,3)$ which (like many other Gepner models) can be related to a sigma model on a Calabi-Yau manifold, namely the quintic hypersurface in $\mathbb{C} P^{4}$. In the rest of the text, we will occasionally abbreviate the models by collecting the $n$ repeated levels together as $k^{n}$. For instance, $\left(k_{1}^{3}, k_{2}\right) \equiv\left(k_{1}, k_{1}, k_{1}, k_{2}\right)=\left(k_{1}, k_{2}, k_{1}, k_{1}\right)=\ldots$. 
The quintic therefore may be written as $\left(3^{5}\right)$. We will also use $c\left(k_{1}, k_{2}, \ldots, k_{r}\right)$ to refer to the $\operatorname{sum} \sum_{i=1}^{r} c\left(k_{i}\right)$.

To ensure spacetime supersymmetry and tachyon-freedom, the tensor product of minimal models and external CFT needs to be subject to certain projections. Most importantly, one demands that the total fermion number of any state be odd (the GSO projection). We can write this in the form

$$
q_{\mathrm{ext}}+q_{\mathrm{int}} \in 2 \mathbb{Z}+1
$$

with the sum of the minimal model charges

$$
q_{\text {int }}=\sum_{j=1}^{r} \frac{m_{j}}{k_{j}+2}-\frac{s_{j}}{2}
$$

and the charge of the external fermions

$$
q_{\mathrm{ext}}=\frac{d}{2} \frac{s_{0}}{2}
$$

here, $s_{0}=-1,0,1,2$ labels the irreducible representations $(c, o, s, v)$ of $\mathrm{SO}(d)_{1}$, such that $q_{\text {ext }}$ simply amounts to the external fermion number. States in the tensor product that do not satisfy (4.8) are projected out.

Apart from this GSO-projection, one also has to ensure that only states in tensor products of $r+1 \mathrm{NS}$ or R sectors are packaged together; this is enforced by removing all states which do not satisfy

$$
\frac{d}{2} \frac{s_{0}}{2}+\frac{s_{j}}{2} \cdot \in \mathbb{Z} .
$$

Projecting out states from a CFT normally spoils modular invariance of the closed string partition function - unless one treats the projection as part of an orbifold procedure (more precisely, in the case of Gepner's construction, a simple current orbifolding). Modular invariance is then restored by adding suitable twisted sectors to the states left from the tensor product theory one started with. We refer to [9] for more details and for explicit expressions of the full partition function.

In order to have a large-volume interpretation of a Gepner model in terms of a sigma model on a Calabi-Yau manifold $[12,13]$ without fluxes, it is mandatory that $c_{\text {int }}$ is a multiple of 3. For the purposes of having a consistent string background, however, this restriction is dispensable. One can instead start from an external SCFT like the linear dilaton for a suitable $\epsilon$ and tensor it with a several $N=2$ minimal models giving the correct value of $c_{\text {int }}$ without violating any of the constraints imposed by string theory, provided that that total central charge of internal and external sum up to 15 ,

$$
c=c_{\mathrm{ext}}+c_{\mathrm{int}}=15 \quad \text { with } \quad c_{\mathrm{ext}}=6-\epsilon \quad \text { and } \quad c_{\mathrm{int}}=9+\epsilon .
$$

In particular, the GSO projection can be performed as usual in a Gepner models, as long as one replaces $q_{\text {ext }}$ from (4.10) by the fermion number counting the $\psi^{\mu}$ from the linear dilaton theory. The condition that only NS sectors or only R sectors are to be tensorised can, of course, also carried over from the $\epsilon=0$ case. Modular invariance will be preserved in the same way as in Gepner models, since the projections can still be understood as orbifoldings. 
In the examples we have studied, spacetime supersymmetry is broken. Apart from that, the main changes when generalising Gepner's construction to non-zero charge deficit are the loss of a geometric picture involving Calabi-Yau manifolds, and very different spectra of massless and light fields, which will be the topic of the next subsection.

First, however, we have to remember that, for $\epsilon<0$, we need to add a brane to our background in order to keep the dilaton from running away to infinity. This brane should fill the external four-dimensional spacetime, i.e. satisfy Neumann boundary conditions on the external bosons. Branes of that type were already studied in [25] and also in [26]. Boundary states for Gepner models were constructed in [23, 24]; the internal part of these takes the form

$$
\left.|\alpha\rangle=\sum_{\boldsymbol{\lambda}, \boldsymbol{\mu}} B_{\alpha}^{\boldsymbol{\lambda}, \boldsymbol{\mu}}|\boldsymbol{\lambda}, \boldsymbol{\mu}\rangle\right\rangle
$$

with

$$
B_{\alpha}^{\boldsymbol{\lambda}, \boldsymbol{\mu}} \sim \prod_{j=1}^{r} \frac{\sin \pi \frac{\left(l_{j}+1\right)\left(L_{j}+1\right)}{k_{j}+2}}{\sin \pi \frac{l_{j}+1}{k_{j}+2}} e^{i \pi m_{j} M_{j} /\left(k_{j}+2\right)} e^{-i \pi s_{j} S_{j} / 2} .
$$

where the $\boldsymbol{\lambda}, \boldsymbol{\mu}$ stand for the collection of $\left(l_{i}, m_{i}, s_{i}\right)$ labels, while $\alpha$ is short for the integer $\left(L_{i}, M_{i}, S_{i}\right)$ labels of the boundary states themselves. These formulas were written with $\epsilon=0$ Gepner models in mind, but with the above adaption of the projections, they work just as well in our more general situation. The brane we require for $\epsilon<0$ can therefore be any (GSO-projected) tensor product of a Neumann brane for the linear dilaton with a boundary state for the internal sector.

There is one further condition we have to keep in mind, namely that the brane we use should not lead to tadpoles for massless RR-fields (tadpoles for massive fields are harmless and are cured by a suitable shift in their vacuum expectation value [14]). Such tadpoles can be cancelled forming suitable (model-dependent) superpositions of the above boundary states (exploiting the fact that the coefficients (4.13) contain roots of unity); cf. the construction of branes carrying torsion charges only for the quintic given in [30]. However, as it turns out, many of the $\epsilon \neq 0$ models we studied so far do not contain any massless RR-fields, so there are no tadpoles to cancel.

\section{Some examples, and spectra of light fields}

In this section, we discuss some specific examples, with special focus on the content of massless and light fields. We will find pronounced differences between cases with nonvanishing central charge deficit and Gepner's original construction.

In a Gepner compactification to $D=4$ dimensions (with $\epsilon=0$, the number $r$ of minimal models used satisfies $3<r \leq 9$ because $1 \leq c\left(k_{i}\right) \leq 3$. The most famous example is $k=(3,3,3,3,3)$ which (like many other Gepner models) can be related to a sigma model on a Calabi-Yau manifold, in this case the quintic hypersurface in $\mathbb{C} P^{4}$. Gepner models typically have quite a large number of massless moduli in the NS-sector, coming from the chiral and anti-chiral primaries. These are $N=2$ primary fields $|\psi\rangle$ obeying $G_{-\frac{1}{2}}^{+}|\psi\rangle=0$ or $G_{-\frac{1}{2}}^{-}|\psi\rangle=0$, and it can be shown that they arise from tensor products of minimal model 
(anti-)chiral fields, labeled $\left(l_{i}, \pm l_{i}, 0\right)$, such that their conformal dimensions sum up to $\frac{1}{2}$. The Gepner model corresponding to the quintic, e.g., has $101+1$ massless moduli of the (chiral,chiral) or (chiral,anti-chiral) type.

Basically, the reason that so many $h_{\text {int }}=\frac{1}{2}$ fields survive the GSO projection is that $c_{\text {int }}=9$ : this forces the levels $k_{i}$ of the constituent minimal models - or more precisely the numbers $k_{i}+2$ - to satisfy conditions on their relative divisibility. The same denominators $k_{i}+2$ from the minimal model central charges also occur in the U(1)-charges $q_{m, s}^{l}$, and due to the tuned divisibility there are many possible choices of $\left(m_{i}, s_{i}\right)$ satisfying the GSO condition. In view of this connection, one might already expect that our epsilon-Gepner models with $c_{\text {int }}=9+\epsilon$ for $\epsilon \neq 0$ will typically have substantially fewer massless modes than Gepner models, or indeed none at all. This expectation is confirmed when studying concrete examples.

Before presenting a few of these, let us make some general observations on tensor products of $N=2$ minimal models with $c_{\text {int }}=9+\epsilon$. To get $0<\epsilon \ll 1$, one only needs to consider models with $r=4, \ldots, 8$ tensor factors; for $r=9$, the smallest possible central charge deficit is $\epsilon=\frac{1}{2}$, for the model with levels $k=\left(1^{8}, 2\right)$.

A small non-zero value of $|\epsilon|$ usually requires at least one of the levels $k_{i}$ to be rather large, which implies that the $i^{\text {th }}$ minimal model contributes a very large number of fields to the tensor product theory; checking the spectrum for light fields is therefore a (somewhat non-trivial) task for the computer.

Having one large $k_{i}$ also means that there is a narrow mass gap $\Delta m^{2} \sim\left(k_{i}+2\right)^{-1}$ above the lowest-lying states. In [3], this was viewed as a problematic issue, as low mass gaps might be interpreted as a signal for large internal "dimensions" of the compactification. However, the same feature occurs in ordinary Gepner models with $c_{\text {int }}=9$. There is no reason to perform a limit $\epsilon \rightarrow 0$, as is hinted at in [3]; rather, $\epsilon$ is a fixed (discrete) input parameter specifying the string background. Still, the narrow mass gaps present the following challenge: For phenomenological applications, one would prefer models with as vast a "desert" above the lowest-mass states as possible, so one is faced with an optimisation problem balancing small $\epsilon$ against large $k_{i}$.

How small one can make $\epsilon$ depends crucially on its sign. For $\epsilon<0$, one can approach $c_{\text {int }}=9$ arbitrarily closely from below: e.g., one can start from a tensor product with central charge 6 (from a K3 Gepner model), then tensor with one further $N=2$ minimal model, with very large $k_{i}$. We will however also present an example manufactured from a $c_{\text {int }}=9$ Gepner model below.

For $\epsilon>0$, the situation is very different. When restricting ourselves to tensor products of $N=2$ minimal models as outlined above, there is actually a smallest possible positive $\epsilon$ that can be achieved. The reason is that the minimal model central charges (4.2) form a discrete series between 1 and 3 . More details on series of $c_{\text {int }}>9$ for our epsilon-Gepner models will be given in the appendix.

The "best" $c_{\text {int }}>9$ model, i.e. the one with the smallest positive $\epsilon$ we can construct in this way, has levels $k=(1,5,41,1805)$ and central charge

$$
c_{\text {int }}=\frac{4895164}{543907}, \quad \epsilon \approx 1.8 \times 10^{-6} .
$$


Using the mass formula (3.10) worked out in the previous section, one can compute the spectrum of tachyonic, massless, and "light" fields in the NS and the R sector for this compactification. One finds

- Tachyons: 0 (NS), 0 (R).

- Massless fields: 1 (NS), 0 (R).

- Massive fields with $m^{2}<\frac{1}{2}$ : 161 (NS), 4 (R).

The single massless state comes from the identity $[(0,0,0), \ldots,(0,0,0)]$ in the internal minimal model and has external oscillator number $N=\frac{1}{2}$, i.e. it gives the graviton. The next lightest field has $[(0,0,0),(0,0,0),(0,0,0),(0,0,0),(2,0,0)]$ and $N=\frac{1}{2}$ and has $m^{2}=$ $2 / 1807 \approx 10^{-3}$, i.e. its mass sits at about 1000 times the scale set by $\epsilon$. The four massive Ramond-Ramond fields listed here are all rather heavy with $m^{2} \sim 1 / 2$. Just as in the model considered in [3], the spectrum has no spacetime supersymmetry.

A comment on finding such spectra is in order. Since our models typically have one level $k_{i}$ which is very large, the tensor product of minimal models contains very many fields — about $10^{12}$ in the above example $k=(1,5,41,1805)$, Sifting through those for suitable $h$-values to identify tachyonic and massless fields takes too long a time even on a computer. Therefore, one first reduces the number of fields by performing the GSO projection (an easier task since it only involves the $m_{i}$ and $s_{i}$ quantum numbers, not the $l_{i}$ ) and then checks the conformal dimensions of the surviving states.

The problem of listing states becomes even more severe for conformal dimensions $h_{\text {int }}>\frac{1}{2}$, as additional $N=1$ primary fields can arise from linear combinations of descendants of $N=2$ primaries. As long as we concentrate on light string modes, we can however ignore those additional states.

We see that our "best" $\epsilon>0$ model has no massless moduli at all. This may be compared to the closely related model $(1,5,41,1804)$, which has $c_{\text {int }}=9$ and a geometric interpretation as a Calabi-Yau sigma model. Here, one finds 504 massless moduli, see [10, 11].

From this Calabi-Yau Gepner model, one can of course also obtain a compactification with small negative central charge deficit: The model with $k=(1,5,41,1803)$ has

$$
c_{\mathrm{int}}=\frac{4889744}{543305}, \quad \epsilon \approx-1.8 \times 10^{-6} .
$$

In this case, one finds the following spectrum of low-lying states:

- Tachyons: 0 (NS), 0 (R).

- Massless fields: 1 (NS), 0 (R).

- Massive fields with $m^{2}<\frac{1}{2}$ : 161 (NS), 0 (R).

Again, the only massless field in the NSNS sector corresponds to the graviton, and the next NS lightest field is still $[(0,0,0),(0,0,0),(0,0,0),(0,0,0),(2,0,0)]$ with $N=\frac{1}{2}$; its mass is $m^{2}=2 / 1805 \approx 10^{-3}$. 
While the numbers of light fields in the NS and R sector depends crucially on the levels $k_{i}$ defining the compactification, we can make a few general remarks, exploiting the GSO projection and familiar bounds on conformal dimensions in $N=2$ SCFTs, namely $h_{\text {int }} \geq$ $\frac{\left|q_{\text {int }}\right|}{2}$ in the NS sector and $h_{\text {int }} \geq \frac{c_{\text {int }}}{24}$ in the $\mathrm{R}$ sector. This allows to show that, with the mass formula (3.10), models with $\epsilon>0$ are tachyon-free and moreover have no massless fields in the Ramond sector. For models with $\epsilon<0$, one can at least show that there are no tachyonic modes in the NS sector. In case all the $k_{i}+2$ are pairwise coprime, one can make some further statements, e.g. concerning absence of R ground states from the GSO-projected theory.

Some further examples of models with small central charge deficits are listed in the appendix, along with counts of the low-lying states.

\section{Conclusions and open problems}

In this paper we have discussed worldsheet CFTs where the split in central charge between the internal and external sector is shifted to $6-\epsilon$ and $9+\epsilon$. This leads to an effective potential for the dilaton which can be stabilised by the addition of suitable D-branes. The result is an effective description, valid for small $\epsilon$ that admits a stable $A d S_{4}$ vacuum with no massless moduli. We presented explicit compact CFTs in the form of epsilon-Gepner models and also some exact D-brane boundary states. In a sense one might think of these epsilon-Gepner models as CFT analogues of Calabi-Yau compactifications with fluxes. Indeed this raises the possibility that in some cases it might be possible to realise a geometrical limit where the epsilon-Gepner models can be related to Calabi-Yau compactifications with fluxes.

Our models in this sense provide a worldsheet analogue of the flux compactification mechanisms that have been studied in supergravity. The advantages of our construction are that the internal CFT can be described exactly and yet one still obtains a spacetime effective picture that is weakly curved and weakly coupled. On the other hand we have not addressed some important questions. These include combining the techniques used here with models that have a realistic spectrum. In addition one would ultimately like to extend the analysis to include a sort of KKLT mechanism [16] whereby the cosmological constant is lifted to a positive value. Our models also naively break supersymmetry, already because the D-branes do not carry a charge (and hence cannot saturate a lower bound on the mass by the charge that is characteristic of supersymmetric D-branes). However it could be possible with more care to obtain supersymmetric vacua, or at least vacua where supersymmetry is broken at a small scale determined by $\epsilon$.

We also saw that there are in fact a large number of such models. Indeed since there are infinitely many models with $\epsilon<0$ if we relax the usual Gepner-model constraint that $\epsilon=0$, it would appear that our models will be rather generic since the separate levels of the minimal models are no longer need to be related by having common factors. In such cases the absence of massless moduli seems to be rather generic (but not universal). We could also use a variety of other CFTs for the internal sector. For example we could consider tensor products of $N=1$ minimal models, which will provide a "denser set" of $\epsilon$-values to choose from, while still retaining rationality. But other options are available, so there is a chance to find models with a more realistic spectrum. In addition, it would be nice to obtain an exact description of the $A d S_{4}$ vacuum at the minimum of the potential 


\section{Acknowledgments}

We would like to thank I. Antoniadis, M. Breuning, I. Brunner, N. Carqueville, R. Helling, W. Lerche and J. Walcher for discussions. This work was supported in part by STFC rolling grant ST/G000395/1.

\section{A 'Best' $\epsilon>0$ models}

In the following, a tensor product of $r$ minimal models with levels $\left(k_{1}, k_{2}, \ldots, k_{r}\right)$ will be written in an ascending order of their levels, i.e., $k_{i-1} \leq k_{i}$. Since $c(k+1)>c(k)$, $c\left(k_{1}^{\prime}, k_{2}^{\prime}, \ldots, k_{r}^{\prime}\right) \leq c\left(k_{1}, k_{2}, \ldots, k_{r}\right)$ for all $k_{i}^{\prime} \leq k_{i}$. The algorithm we use finds the first $\left(k_{1}, k_{2}, \ldots, k_{r}\right),\left(k_{i-1} \leq k_{i}\right)$ model with central change $9+\epsilon, \epsilon>0$, i.e.,

$$
c\left(k_{1}, k_{2}, \ldots, k_{r}-1\right) \leq 9 \quad \text { and } \quad c\left(k_{1}, k_{2}, \ldots, k_{r}\right)>9 .
$$

Each subsequent model $\left(k_{1}, k_{2}, \ldots, k_{r}+1\right)$ will have a central charge greater than $9+\epsilon$. Given an upperbound $\epsilon^{\prime}$, the algorithm also determines the model $\left(k_{1}, k_{2}, \ldots, k_{r-1}, k_{r}^{\prime}\right)$ with highest $k_{r}^{\prime}$ such that $c\left(k_{1}, k_{2}, \ldots, k_{r}^{\prime}\right) \leq 9+\epsilon^{\prime}$.

In the following table, we list models with $0<\epsilon \leq 0.05$. The total number of models in this range is infinite and we list a few series of models from $\left(k_{1}, k_{2}, \ldots, k_{r}\right)$ to $\left(k_{1}, k_{2}, \ldots, k_{r}^{\prime}\right)$ as defined above. We find the total number of these series to be 160 .

\begin{tabular}{|c|c|c|c|c|}
\hline$r$ & $\left(k_{1}, k_{2}, \ldots, k_{r}\right)$ & $c$ & $\epsilon$ & $\left(k_{1}, k_{2}, \ldots, k_{r}^{\prime}\right)$ \\
\hline 4 & $(1,5,41,1805)$ & $4895164 / 543907$ & $1 / 543907 \sim 1.84 \times 10^{-6}$ & $(1,5,41, \infty)$ \\
& $(1,5,42,923)$ & $1282051 / 142450$ & $1 / 142450 \sim 7 \times 10^{-6}$ & $(1,5,42, \infty)$ \\
& $(1,5,45,393)$ & $1169596 / 129955$ & $1 / 129955 \sim 7.7 \times 10^{-6}$ & $(1,5,45, \infty)$ \\
& $(1,5,43,629)$ & $596296 / 66255$ & $1 / 66255 \sim 1.5 \times 10^{-5}$ & $(1,5,43, \infty)$ \\
& $(1,5,44,482)$ & $350659 / 38962$ & $1 / 38962 \sim 2.5 \times 10^{-5}$ & $(1,5,44, \infty)$ \\
& $(2,3,19,419)$ & $265231 / 29470$ & $1 / 29470 \sim 3.4 \times 10^{-5}$ & $(1,5,19, \infty)$ \\
& $(3,3,9,109)$ & $18316 / 2035$ & $1 / 2035 \sim 4.9 \times 10^{-4}$ & $(3,3,9,1318)$ \\
& $(4,4,5,41)$ & $2710 / 301$ & $1 / 301 \sim 3.32 \times 10^{-3}$ & $(4,4,5,62)$ \\
& $(1,5,86,86)$ & $1387 / 154$ & $1 / 154 \sim 6.5 \times 10^{-3}$ & $(1,5,86,241)$ \\
& $(1,5,87,87)$ & $5612 / 623$ & $5 / 623 \sim 8.03 \times 10^{-3}$ & $(1,5,87,233)$ \\
& $(5,5,7,8)$ & $947 / 105$ & $2 / 105 \sim 1.9 \times 10^{-2}$ & $(5,5,7,8)$ \\
\hline 5 & $(1,1,2,11,155)$ & $36739 / 4082$ & $1 / 4082 \sim 2.45 \times 10^{-4}$ & $(1,1,2,11, \infty)$ \\
& $(1,1,2,15,39)$ & $12547 / 1394$ & $1 / 1394 \sim 7.2 \times 10^{-4}$ & $(1,1,2,15,59)$ \\
& $(1,1,2,12,83)$ & $10711 / 1190$ & $1 / 1190 \sim 8.4 \times 10^{-4}$ & $(1,1,2,12,278)$ \\
& $(1,1,2,13,59)$ & $5491 / 610$ & $1 / 610 \sim 1.64 \times 10^{-3}$ & $(1,1,2,13,118)$ \\
& $(2,2,2,4,11)$ & $235 / 26$ & $1 / 26 \sim 3.85 \times 10^{-2}$ & $(2,2,2,4,11)$ \\
\hline 6 & $(1,1,1,1,5,41)$ & $2710 / 301$ & $1 / 301 \sim 3.32 \times 10^{-3}$ & $(1,1,1,1,5,62)$ \\
& $(1,1,1,1,6,23)$ & $901 / 100$ & $1 / 100=10^{-2}$ & $(1,1,1,1,6,28)$ \\
& $(1,1,1,1,7,17)$ & $514 / 57$ & $1 / 57 \sim 1.75 \times 10^{-2}$ & $(1,1,1,1,7,19)$ \\
\hline 7 & $(1,1,1,1,1,2,11)$ & $235 / 26$ & $1 / 26 \sim 3.85 \times 10^{-2}$ & $(1,1,1,1,1,2,11)$ \\
& $(1,1,1,1,1,3,6)$ & $181 / 20$ & $1 / 20=5 \times 10^{-2}$ & $(1,1,1,1,1,3,6)$ \\
\hline
\end{tabular}

Table 1. Best $\epsilon>0$ models. 
Note that the model $(1,5,87,86)$ has a smaller $\epsilon$ than that of $(1,5,87,87)$ but since we order models in ascending order of their levels, $(1,5,87,86)$ is included in the $(1,5,86,86)$ to $(1,5,86,241)$ series. Also note that there is no $r=8$ model with $0<\epsilon \leq 0.05$ as the first model after $c\left(1^{7}, 4\right)=9$ gives $c\left(1^{7}, 5\right) \sim 9.14$.

The first model in the table $(1,5,41,1805)$ has the smallest possible positive $\epsilon \sim 1.84 \times$ $10^{-6}$. Note that $c(1,5,41,1804)=9$. The fact that $(1,5,41,1805)$ has the smallest $\epsilon$ can be shown by starting with the model $\left(1^{4}\right)=\left(k_{1}=1, k_{2}=1, k_{3}=1, k_{4}=1\right)$ and incrementing $k_{i}$ one at a time resulting in the smallest step increase in the central charge. If $k_{i}, i=1,2,3$ are kept fixed at 1 and $k_{4}$ is increased, we will never obtain a total central charge of 9 . Hence we find a bound $k_{3}>1$. By induction on this argument, we see that at least $3 k_{i}$ 's must be greater than 1 . We repeat this procedure until the combination $(1,5,41,1804)$ is obtained with central charge 9 and the very next model $(1,5,41,1805)$ provides us with the smallest possible change in the central charge over 9 . We can also see that there are infinite number of $r=4, c \leq 9.05$ models with three levels $k_{1}=1, k_{2}=5, k_{3}=41$ by observing that $c(1,5,41)<6.05$ and $c(k)$ is bounded from above by 3 .

\section{B Examples of models}

Here we summarise data calculated for a few example models. The mass scale for light fields is set to $M^{2}=1000 \epsilon$ in all cases below.

\begin{tabular}{|c|c|c|c|c|c|}
\hline $\begin{array}{c}\text { Levels } \\
k_{i}\end{array}$ & $\begin{array}{c}\text { Central } \\
\text { charge } c\end{array}$ & $\begin{array}{c}\text { Charge } \\
\text { deficit } \epsilon\end{array}$ & $\begin{array}{c}\text { Tach- } \\
\text { yons }\end{array}$ & $\begin{array}{c}\text { Massless } \\
\text { fields }\end{array}$ & $\begin{array}{c}\text { Light fields } \\
\left(m^{2} \leq M^{2}\right)\end{array}$ \\
\hline$(1,5,41,1805)$ & $4895164 / 543907$ & $1.84 \times 10^{-6}$ & 0 & $1(\mathrm{NS}), 0(\mathrm{R})$ & $1(\mathrm{NS}), 0(\mathrm{R})$ \\
$(1,5,41,1806)$ & $2448937 / 272104$ & $3.68 \times 10^{-6}$ & 0 & $1(\mathrm{NS}), 0(\mathrm{R})$ & $2(\mathrm{NS}), 0(\mathrm{R})$ \\
$(1,5,42,923)$ & $1282051 / 142450$ & $7 \times 10^{-6}$ & 0 & $1(\mathrm{NS}), 0(\mathrm{R})$ & $2(\mathrm{NS}), 0(\mathrm{R})$ \\
$(1,5,45,393)$ & $1169596 / 129955$ & $7.7 \times 10^{-6}$ & 0 & $1(\mathrm{NS}), 0(\mathrm{R})$ & $1(\mathrm{NS}), 0(\mathrm{R})$ \\
$(1,5,43,629)$ & $596296 / 66255$ & $1.5 \times 10^{-5}$ & 0 & $2(\mathrm{NS}), 0(\mathrm{R})$ & $4(\mathrm{NS}), 0(\mathrm{R})$ \\
$(1,5,44,482)$ & $350659 / 38962$ & $2.5 \times 10^{-5}$ & 0 & $2(\mathrm{NS}), 0(\mathrm{R})$ & $3(\mathrm{NS}), 0(\mathrm{R})$ \\
\hline
\end{tabular}

Table 2. Spectrum of some $\epsilon>0$ models.

\begin{tabular}{|c|c|c|c|c|c|}
\hline $\begin{array}{c}\text { Levels } \\
k_{i}\end{array}$ & $\begin{array}{c}\text { Central } \\
\text { charge } c\end{array}$ & $\begin{array}{c}\text { Charge } \\
\text { deficit } \epsilon\end{array}$ & $\begin{array}{c}\text { Tach- } \\
\text { yons }\end{array}$ & $\begin{array}{c}\text { Massless } \\
\text { fields }\end{array}$ & $\begin{array}{c}\text { Light fields } \\
\left(m^{2} \leq M^{2}\right)\end{array}$ \\
\hline$(1,5,41,1803)$ & $4889744 / 543305$ & $-1.84 \times 10^{-6}$ & 0 & $1(\mathrm{NS}), 0(\mathrm{R})$ & $1(\mathrm{NS}), 0(\mathrm{R})$ \\
$(1,5,41,1802)$ & $2443517 / 271502$ & $-3.68 \times 10^{-6}$ & 0 & $1(\mathrm{NS}), 0(\mathrm{R})$ & $2(\mathrm{NS}), 0(\mathrm{R})$ \\
$(1,5,42,921)$ & $1279277 / 142142$ & $-7.04 \times 10^{-6}$ & 0 & $1(\mathrm{NS}), 0(\mathrm{R})$ & $2(\mathrm{NS}), 0(\mathrm{R})$ \\
$(1,5,43,627)$ & $594404 / 66045$ & $-1.51 \times 10^{-5}$ & 0 & $2(\mathrm{NS}), 0(\mathrm{R})$ & $4(\mathrm{NS}), 0(\mathrm{R})$ \\
$(1,5,44,480)$ & $349208 / 38801$ & $-2.58 \times 10^{-5}$ & 0 & $2(\mathrm{NS}), 0(\mathrm{R})$ & $3(\mathrm{NS}), 0(\mathrm{R})$ \\
$(1,5,45,392)$ & $583315 / 64813$ & $-3.1 \times 10^{-5}$ & 0 & $1(\mathrm{NS}), 0(\mathrm{R})$ & $3(\mathrm{NS}), 0(\mathrm{R})$ \\
\hline
\end{tabular}

Table 3. Spectrum of some $\epsilon<0$ models. 
Open Access. This article is distributed under the terms of the Creative Commons Attribution Noncommercial License which permits any noncommercial use, distribution, and reproduction in any medium, provided the original author(s) and source are credited.

\section{References}

[1] R.C. Myers, New dimensions for old strings, Phys. Lett. B 199 (1987) 371 [SPIRES].

[2] C.G. Callan Jr., E.J. Martinec, M.J. Perry and D. Friedan, Strings in background fields, Nucl. Phys. B 262 (1985) 593 [SPIRES].

[3] S.P. de Alwis, J. Polchinski and R. Schimmrigk, Heterotic strings with tree level cosmological constant, Phys. Lett. B 218 (1989) 449 [SPIRES].

[4] A.H. Chamseddine, A study of noncritical strings in arbitrary dimensions, Nucl. Phys. B 368 (1992) 98 [SPIRES].

[5] I. Antoniadis, C. Bachas, J.R. Ellis and D.V. Nanopoulos, Cosmological string theories and discrete inflation, Phys. Lett. B 211 (1988) 393 [SPIRES].

[6] I. Antoniadis, C. Bachas, J.R. Ellis and D.V. Nanopoulos, An expanding universe in string theory, Nucl. Phys. B 328 (1989) 117 [SPIRES].

[7] V.S. Dotsenko and V.A. Fateev, Conformal algebra and multipoint correlation functions in $2 D$ statistical models, Nucl. Phys. B 240 (1984) 312 [SPIRES].

[8] V.S. Dotsenko and V.A. Fateev, Four point correlation functions and the operator algebra in the two-dimensional conformal invariant theories with the central charge $c<1$, Nucl. Phys. B 251 (1985) 691 [SPIRES].

[9] D. Gepner, Space-time supersymmetry in compactified string theory and superconformal models, Nucl. Phys. B 296 (1988) 757 [SPIRES].

[10] M. Lynker and R. Schimmrigk, ADE quantum Calabi-Yau manifolds, Nucl. Phys. B 339 (1990) 121 [SPIRES].

[11] J. Fuchs, A. Klemm, C. Scheich and M.G. Schmidt, Gepner models with arbitrary affine invariants and the associated Calabi-Yau spaces, Phys. Lett. B 232 (1989) 317 [SPIRES].

[12] B.R. Greene, String theory on Calabi-Yau manifolds, hep-th/9702155 [SPIRES].

[13] E. Witten, Phases of $N=2$ theories in two dimensions, Nucl. Phys. B 403 (1993) 159 [hep-th/9301042] [SPIRES].

[14] M. Dine, N. Seiberg, X.G. Wen and E. Witten, Nonperturbative effects on the string world sheet. 2, Nucl. Phys. B 289 (1987) 319 [SPIRES].

[15] K. Becker, M. Becker, C. Vafa and J. Walcher, Moduli stabilization in non-geometric backgrounds, Nucl. Phys. B 770 (2007) 1 [hep-th/0611001] [SPIRES].

[16] S. Kachru, R. Kallosh, A.D. Linde and S.P. Trivedi, De Sitter vacua in string theory, Phys. Rev. D 68 (2003) 046005 [hep-th/0301240] [SPIRES].

[17] S. Kachru et al., Towards inflation in string theory, JCAP 10 (2003) 013 [hep-th/0308055] [SPIRES].

[18] M.R. Douglas and S. Kachru, Flux compactification, Rev. Mod. Phys. 79 (2007) 733 [hep-th/0610102] [SPIRES]. 
$[19]$ T.R. Taylor and C. Vafa, $R R$ flux on Calabi-Yau and partial supersymmetry breaking, Phys. Lett. B 474 (2000) 130 [hep-th/9912152] [SPIRES].

[20] J. Polchinski, String theory. Vol. 1: an introduction to the bosonic string, Cambridge Univ. Pr., Cambridge U.K. (1998) [SPIRES].

[21] J. Polchinski, String theory. Vol. 2: superstring theory and beyond, Cambridge Univ. Pr., Cambridge U.K. (1998) [SPIRES].

[22] P.K. Townsend, Quintessence from M-theory, JHEP 0111 (2001) 042 [hep-th/0110072] [SPIRES].

[23] A. Recknagel and V. Schomerus, D-branes in Gepner models, Nucl. Phys. B 531 (1998) 185 [hep-th/9712186] [SPIRES].

[24] A. Recknagel, Permutation branes, JHEP 04 (2003) 041 [hep-th/0208119] [SPIRES].

[25] M. Bershadsky and D. Kutasov, Open string theory in $(1+1)$-dimensions, Phys. Lett. B 274 (1992) 331 [hep-th/9110034] [SPIRES].

[26] M. Li, Dirichlet boundary state in linear dilaton background, Phys. Rev. D 54 (1996) 1644 [hep-th/9512042] [SPIRES].

[27] M. Frau, I. Pesando, S. Sciuto, A. Lerda and R. Russo, Scattering of closed strings from many D-branes, Phys. Lett. B 400 (1997) 52 [hep-th/9702037] [SPIRES].

[28] M. Billó, P. Di Vecchia and D. Cangemi, Boundary states for moving D-branes, Phys. Lett. B 400 (1997) 63 [hep-th/9701190] [SPIRES].

[29] I. Brunner, K. Hori, K. Hosomichi and J. Walcher, Orientifolds of Gepner models, JHEP 02 (2007) 001 [hep-th/0401137] [SPIRES].

[30] I. Brunner and J. Distler, Torsion D-branes in nongeometrical phases, Adv. Theor. Math. Phys. 5 (2002) 265 [hep-th/0102018] [SPIRES].

[31] I. Antoniadis, J.-P. Derendinger and T. Maillard, Nonlinear $N=2$ supersymmetry, effective actions and moduli stabilization, Nucl. Phys. B 808 (2009) 53 [arXiv:0804.1738] [SPIRES]. 